\title{
Les déterminants du risque de liquidité dans les banques islamiques de la région MENA : Une analyse par la méthode des données de panel
}

\author{
Tarik QUAMAR ${ }^{1}$, Ghalia BENAACHIR ${ }^{2}$ \\ ${ }^{l}$ FSJES Ain Chock, Université Hassan II, Laboratoire de recherche : Finance, Banque et Gestion des Risques, Casablanca, \\ Maroc \\ ${ }^{2}$ FSJES Ain Chock, Université Hassan II, Laboratoire de recherche : Finance, Banque et Gestion des Risques, Casablanca, \\ Maroc
}

\begin{abstract}
Résumé : Le présent article vise à analyser les déterminants du risque de liquidité au sein de 62 banques islamiques de 10 pays de la région MENA sur une période qui s'étale entre 2011 et 2019. Pour y parvenir, nous avons eu recours à la méthode des moments généralisés (GMM) appliquée sur un panel dynamique non cylindré. Les résultats empiriques démontrent que le risque de crédit, la marge nette d'intérêt, la taille de la banque et l'inflation impactent négativement le risque de liquidité à long terme tandis que le ratio du rendement des actifs l'influence positivement. Nous avons aussi constaté que le ratio d'adéquation des fonds propres, l'inflation et la taille de la banque influencent positivement le risque de liquidité à court terme, alors que ce dernier est influencé de façon négative par le ratio du rendement des actifs.
\end{abstract}

Mots-clés : Banque islamique, Liquidité, Risque de liquidité, Données de panel.

\section{Introduction}

Depuis la crise financière des subprimes, la gestion de la liquidité ne cesse de constituer un sujet qui revêt un intérêt grandissant dans la littérature financière. En effet, dans les institutions financières, notamment les banques, la liquidité est d'une importance fondamentale pour le développement de leurs activités étant donné que la pierre angulaire de leur modèle économique est la transformation des fonds levés auprès des déposants en crédits à accorder à leurs clients, impliquant parfois une discordance entre l'échéance des actifs et celle des passifs.

Ainsi, le risque de liquidité résulte d'une inadéquation entre l'offre et la demande de fonds. La différence entre ces dernières est appelée «position de liquidité nette » que les banques doivent gérer avec précaution afin de réduire leur risque de liquidité (Alzoubi, 2017). Pour y parvenir, les banques classiques peuvent recourir à différents mécanismes et outils de gestion alors que les banques islamiques doivent faire plus en se conformant aux restrictions imposées par les préceptes de la charia, ce qui rend plus complexe la gestion de leur risque de liquidité. La gestion dudit risque de liquidité constitue, sans équivoque, un défi majeur des politiques de gestion des risques au sein des banques islamiques, d'où l'importance de l'analyse de ce risque et des facteurs qui peuvent l'influencer.

Par ailleurs, la banque islamique cherche à optimiser la relation liquidité-rentabilité grâce à un compromis entre la liquidité et le rendement. A cet égard, une gestion efficace de la liquidité permet de 
réduire le coût de l'intermédiation et améliore subséquemment la rentabilité bancaire. Théoriquement, la banque devrait détenir un niveau de liquidité « suffisant » pour se prémunir contre le risque de liquidité (Diamond et Dybvig, 1983). Or, la détermination d'un tel niveau constitue même le socle de la gestion du risque de liquidité bancaire et obéit à différentes contraintes d'ordre prudentiel, stratégique et opérationnel. Par conséquent, il est essentiel d'identifier les principaux déterminants du risque de liquidité dans les banques islamiques afin d'en améliorer la gestion. Dès lors, cet article vise à analyser empiriquement les facteurs qui influencent le risque de liquidité inhérent aux banques islamiques sur la période 2011-2019 en exploitant une base de données de panel relative à un échantillon composé de 62 banques islamiques de la région MENA en ayant recours à la méthode des moments généralisés (GMM).

Ce travail de recherche est scindé en deux parties. La première présente une revue de la littérature empirique relative aux déterminants du risque de liquidité dans un contexte bancaire islamique. La seconde partie, quant à elle, expose la méthodologie de recherche adoptée ainsi qu'une discussion des résultats obtenus.

\section{Le risque de liquidité dans les banques islamiques : une revue de littérature}

Selon les principes directeurs de la gestion des risques de l'IFSB (Islamic Financial Services Board), le risque de liquidité constitue la perte potentielle qu'encourent les banques islamiques résultant de leur incapacité de remplir leurs obligations à temps ou de financer l'augmentation des actifs sans encourir des pertes inacceptables. C'est ainsi que l'une des plus grandes priorités d'une institution financière islamique est de maintenir une position de liquidité suffisante du fait que le risque de liquidité peut s'avérer critique, contagieux et générateur d'autres risques bancaires. A ce titre, sa gestion est devenue d'un intérêt vital pour la stabilité de l'ensemble du système bancaire (Syed, 2013). Ainsi, la mauvaise gestion des fonds et la volatilité des retraits des déposants figurent parmi les principales causes des problèmes de liquidité chez les banques islamiques où le risque de liquidité peut se présenter sous différentes formes : un excès de liquidité, une insuffisance d'actifs liquides permettant de couvrir les engagements du passif ou encore un coût élevé de mobilisation des fonds. La banque islamique doit donc chercher une position optimale de liquidité à laquelle fait référence un adage du milieu bancaire : « une insuffisance de liquidité peut tuer une banque rapidement, tandis qu'un excès de liquidité peut la tuer lentement » (Armstrong et Caldwell, 2008).

En effet, l'exposition accrue des banques islamiques au risque de liquidité est principalement due aux faiblesses structurelles caractérisant le système financier islamique dans lequel ces institutions financières évoluent (Al-Muharrami et Hardy, 2013), car souffrant du sous-développement, voire de l'inexistence des marchés monétaires et interbancaires islamiques (Hesse et al., 2008). Dans un tel contexte, la liquidité des banques islamiques est tributaire de la transformation des échéances qui revient à prêter à long terme et emprunter à court terme, d'où une exposition au risque de maturité (Hassoune, 2003). Ce risque est accentué par une présence plus importante sur le marché des particuliers par rapport à celui des entreprises faisant que la banque islamique dispose d'une part plus élevée de dépôts à court terme. Cette analyse a été vérifiée à travers une étude effectuée sur 17 banques islamiques indonésiennes qui a démontré que la plupart des échéances de leurs dépôts ne dépassaient pas un mois (Ismal, 2010). Par ailleurs, la banque islamique, de par sa nature, est confrontée à l'interdiction par la charia des instruments de taux pouvant être utilisés dans la gestion du risque de liquidité. De plus, et puisque la plupart des outils et des emprunts de la Banque Centrale (prêteur en dernier ressort) ne sont pas sharia compliant, la banque islamique est souvent contrainte de conserver 
d'importants niveaux de liquidité afin de faire face aux aléas du marché (Khan et Ahmed, 2001), d'où le problème de liquidité oisive comportant un coût d'opportunité pour la banque islamique à cause de leur faible rendement.

La liquidité bancaire est influencée par de nombreux facteurs internes et externes, et contrairement à la littérature conventionnelle, il existe peu d'études empiriques consacrées au risque de liquidité dans les banques islamiques. De plus, les recherches antérieures portant sur ces dernières se sont soldées par des conclusions différentes. A cet effet, deux études menées respectivement par Akhtar et al. (2011) (étude effectuée sur six banques conventionnelles et six banques islamiques entre 2006 et 2009) et Iqbal (2012) (étude effectuée sur cinq banques conventionnelles et cinq banques islamiques entre 2007 et 2010), ont examiné la gestion du risque de liquidité à travers une analyse comparative de banques islamiques et conventionnelles au Pakistan [(Akhtar et al, 2011); (Iqbal, 2012)] et ont approximé le risque de liquidité par le ratio trésorerie / total des actifs. L'étude empirique de Akhtar et al (2011) a montré que les banques conventionnelles réussissent à assurer une meilleure gestion des ratios de rentabilité et du risque de liquidité par rapport à leurs consœurs islamiques. En revanche, Iqbal (2012) a fait valoir que la position de liquidité dans les banques islamiques est meilleure que celle affichée par les banques conventionnelles.

Dans le même ordre d'idées, Ghenimi et Omri (2015) ont effectué une étude basée sur des données de panel en recourant à la méthode des moindres carrés et les modèles à effets fixes et à effets aléatoires. Cette étude ayant pour finalité d'évaluer l'influence de certains facteurs (CAR, NPL, ROA, ROE, NIM, taille, inflation, PIB) sur le risque de liquidité couvrant 44 banques de la région du Golfe (dont 33 banques conventionnelles et 11 banques islamiques) pour la période 2006 - 2013. Les auteurs ont conclu que les banques islamiques sont plus sensibles à ces facteurs que les banques conventionnelles.

De leur part, Gafrej et Boujelbene (2017) ont exploré l'influence de déterminants aussi bien internes que macroéconomiques sur la liquidité de 86 banques islamiques et 110 banques conventionnelles dans 20 pays différents sur la période allant de 2007 à 2013. Deux mesures ont été utilisées comme indicateurs de la liquidité bancaire, à savoir le ratio actifs liquides / total des actifs et le ratio des actifs liquides rapportés à la somme des dépôts et du financement à court terme. L'étude a conclu que les banques islamiques détiennent davantage de liquidités ce qui leur permet de mieux absorber les retraits massifs de fonds comparativement aux banques conventionnelles. Les auteurs ont également soulevé que la liquidité dans les banques conventionnelles est plus sensible aux facteurs bancaires internes.

Par ailleurs, Boukhatem et Djelassi (2020) ont étudié le secteur bancaire saoudien entre 2008 et 2018 , et ce, en comparant les banques islamiques avec les banques conventionnelles en matière de gestion du risque de liquidité. Le principal constat de ces auteurs fut que les banques islamiques ont tendance à détenir plus d'actifs liquides que les banques conventionnelles, ce qui leur permet d'assurer une meilleure gestion de leur risque de liquidité.

Sur un échantillon de 145 banques, Mohammed et al. (2020) ont examiné l'exposition au risque de liquidité au niveau des banques islamiques en les comparant à des banques conventionnelles et hybrides. Pour y arriver, les auteurs ont fait appel à un modèle à effets aléatoires basé sur des données de panel et ayant pour objectif d'analyser les déterminants du risque de liquidité au sein des différentes catégories de banques objet de l'étude (facteurs bancaires, macroéconomiques, de gouvernance et de structure de propriété). Les résultats ont démontré que les banques islamiques sont les plus exposées au risque de liquidité. 
Dans le cadre des études transnationales, Alzoubi (2017) a analysé les déterminants du risque de liquidité au niveau de 42 banques islamiques issues de 15 pays sur une période allant de 2007 à 2014. L'auteur a appliqué une régression basée sur des données de panel pour examiner l'impact de certains facteurs sur le risque de liquidité approximé par le total des dépôts rapporté au total des actifs. Les résultats ont démontré que la taille de la banque a une relation négative avec le risque de liquidité, du fait que les grandes banques ont tendance à être plus stables, attirant ainsi davantage de dépôts.

Par ailleurs, en se basant sur une régression de données de panel dynamique basée sur la méthode des moments généralisés, Ghenimi et Omri (2018) ont étudié les facteurs impactant le risque de liquidité au niveau de 25 banques islamiques entre 2006 et 2014 et ont démontré que le risque de liquidité est positivement et significativement lié à la taille et au risque de crédit. A son tour, Al Harbi (2020) a examiné les déterminants de la liquidité au sein de 110 banques islamiques entre 1989 et 2008. Les résultats de cette étude ont montré que tous les déterminants adoptés par l'auteur ont une influence statistiquement significative sur la liquidité des banques islamiques de l'échantillon étudié (Al-Harbi, 2020).

Certaines études ont été menées pour examiner le risque de liquidité dans des banques appartenant à un seul pays. C'est ainsi qu'en utilisant une régression linéaire basée sur la méthode des moindres carrés ordinaires, Abdullah et Khan (2012) ont réussi à dresser une analyse des déterminants du risque de liquidité au niveau de banques pakistanaises et étrangères installées au Pakistan. Sur la base des conclusions tirées par ces auteurs, il a été recommandé de minimiser le risque de liquidité dans les banques pakistanaises en augmentant la taille des banques nationale. Toujours pour le cas du Pakistan, Ramzan et Zafar (2014) ont étudié la gestion du risque de liquidité dans les banques islamiques au cours de la période 2007-2011 en approximant le risque de liquidité par le ratio trésorerie / total des actifs. Ces auteurs ont opté pour deux méthodes à savoir, les moindres carrés ordinaires et les modèles à effets fixes et à effets aléatoires et ont pu démontrer que la taille de la banque a une influence positive sur son risque de liquidité.

Dans le même ordre d'idées, Yaacob et al. (2016) ont effectué une étude portant sur le secteur bancaire islamique en Malaisie, dont l'échantillon est composé de 17 banques islamiques. Cette analyse, qui couvre la période s'étalant entre 2000 à 2013, examine les déterminants du risque de liquidité approximé par le LCR et le NSFR en faisant appel à deux groupes de variables : des variables microéconomiques telles que la taille, le ratio CAR, la rentabilité et la qualité des actifs, et des variables macroéconomiques à savoir, le PIB et le taux d'inflation. Les auteurs ont ainsi conclu que les variables macroéconomiques ont une influence plus significative sur le risque de liquidité.

A leur tour, Zaghdoudi et Hakimi (2017) ont testé un échantillon de dix banques tunisiennes au cours de la période de 1980 à 2015 sur la base d'une analyse de données de panel pour identifier les facteurs clés impactant le risque de liquidité bancaire. Ce dernier a été approximé par le ratio des prêts / total des dépôts, tandis que les variables explicatives adoptées sont le risque de crédit, la taille de la banque, le ratio d'adéquation des fonds propres, la croissance économique et le taux d'inflation. Les résultats de l'étude ont montré que les principaux déterminants du risque de liquidité sont en grande partie les facteurs spécifiques à la banque et qu'une petite taille exposerait les banques tunisiennes à un risque de liquidité plus élevé en raison du faible taux de surveillance (Zaghdoudi and Hakimi, 2017).

De leur côté, Shamas et al. (2018) ont étudié les déterminants du risque de liquidité dans 7 banques islamiques du Bahreïn au cours de la période 2007-2011, et ce à l'aide d'une analyse de données de panel. Toutefois, la principale limite de cette étude réside dans le fait qu'elle se soit concentrée 
uniquement sur les facteurs spécifiques aux banques sans tenir compte des facteurs macroéconomiques. De plus, Chowdhury et al. (2019) ont mené une étude, entre 2012 et 2016, sur la relation entre les facteurs spécifiques à la banque et le risque de liquidité au niveau de six banques islamiques au Bangladesh. Les facteurs spécifiques à la banque employés par les auteurs sont la taille, le NPL, le ROA, le ROE, le CAR et le ratio investissement / total dépôts (ITD) et la variable dépendante a été approximée par le ratio actifs liquides / total des actifs. A l'issue de cette analyse, les auteurs ont recommandé aux banques islamiques de diversifier leurs sources de financement et ont reconnu la nécessité de concevoir des titres compatibles avec la charia pour fournir davantage de liquidité au marché.

Mennawi et Ahmed (2020) ont étudié, au cours de la période 2012-2018, les déterminants du risque de liquidité en se basant sur un échantillon de 11 banques islamiques soudanaises. Parmi les variables explicatives utilisées par les auteurs nous retrouvons la trésorerie (CASH), l'investissement en titres à court terme (SECA), le risque de crédit (NPL), en plus d'un facteur qualifié d'ordre macroéconomique qui est le produit intérieur brut (PIB).

A l'issue de cette revue de littérature, nous avons constaté que les études citées, ci-dessus, ont abouti à des résultats divergents, ce qui met en avant l'utilité d'approfondir les analyses se rapportant aux déterminants du risque de liquidité au niveau des banques islamiques sur des périodes plus récentes.

\section{Présentation des déterminants du risque de liquidité bancaire et des hypothèses à tester}

A ce stade de notre analyse, nous exposerons, selon leur nature, les déterminants du risque de liquidité au sein des banques islamiques et qui serviront par la suite de variables explicatives au niveau de notre modèle. Nous retrouvons ainsi les déterminants internes (facteurs spécifiques à la banque) qui sont liés aux décisions de gestion et aux ratios issus des états financiers de la banque, et les déterminants externes (facteurs macroéconomiques) qui sont liés à l'environnement économique où évolue la banque. Cette présentation servira également à construire les hypothèses visant à examiner l'impact de chacun de ces déterminants sur le risque de liquidité dans les banques islamiques de notre échantillon.

\subsection{Les déterminants internes du risque de liquidité bancaire}

\subsubsection{La qualité des actifs}

La qualité des actifs renseigne sur la capacité de la banque à gérer ses actifs, qui constituent une source de revenus servant à financer les opérations bancaires (Menicucci et Paolucci, 2016). C'est une composante permettant de mesurer la solidité financière d'une banque et d'appréhender son risque de crédit. Cette variable est liée à la qualité des prêts accordés par la banque et peut ainsi être approximée par le ratio des prêts non performants (NPL) mesuré par le rapport entre les prêts non performants ou improductifs et le total des prêts (Ghenimi et Omri, 2018; Iqbal, 2012). Certains auteurs ont constaté à travers les résultats de leurs études que l'augmentation du ratio NPL affecte négativement la confiance des déposants, conduit à un accroissement au niveau des retraits et, par conséquent, accentue le risque de liquidité (Alzoubi, 2017; Effendi et Disman, 2017; Ghenimi et Omri, 2018; Incekara et Cetinkaya, 2019; Mennawi et Ahmed, 2020; Mohammad et al., 2020; Zaghdoudi et Hakimi, 2017). Sur la base de ces études, la première hypothèse de cette étude peut être définie comme suit :

H1: La qualité des actifs aura un impact positif sur le risque de liquidité des banques islamiques. 


\subsubsection{La capitalisation bancaire}

Le ratio d'adéquation des fonds propres (CAR) est un indicateur du niveau des fonds propres dans une banque. Deux hypothèses sont retenues pour expliquer la relation entre le ratio CAR d'une banque et sa liquidité. La première suppose que le niveau du capital a un impact négatif sur le risque de liquidité. Pour Menicucci et Paolucci (2016), un ratio d'adéquation des fonds propres élevé est un bon indicateur de la stabilité et de la liquidité d'une banque, ce qui suppose un risque de liquidité relativement maitrisé (Menicucci et Paolucci, 2016). Repullo (2004) a confirmé ce principe en indiquant que l'augmentation du niveau de capital peut conduire les banques à augmenter leur niveau de liquidité en vue d'absorber leur risque de liquidité (Repullo, 2004). De nombreuses études ont confirmé cette hypothèse en indiquant que le ratio CAR affecte négativement le risque de liquidité dans les banques islamiques (Ben Jedidia et Hamza, 2015; Mohammad et al., 2020; Muharam et Kurnia, 2012; Shamas et al., 2018; Yaacob et al., 2016). Sur la base de la discussion ci-dessus, la deuxième hypothèse de l'étude est définie comme suit:

\section{H2 : Le ratio d'adéquation des fonds propres aura un impact négatif sur le risque de liquidité des banques islamiques.}

\subsubsection{La rentabilité}

La rentabilité est un indicateur subjectif de la manière dont une institution pourrait utiliser les actifs liés à son activité principale en vue de générer des bénéfices (Greenwood et Jovanovic, 1990). Il s'agit d'une mesure générale de la santé financière d'un établissement sur une période donnée. La relation entre la rentabilité et la liquidité de la banque a fait l'objet de plusieurs travaux de recherche où la rentabilité bancaire est généralement mesurée par le rendement des capitaux propres (Return On Equity - ROE), le rendement des actifs (Return On Asset - ROA) et la marge nette d'intérêt (Net Interest Margin - NIM).

\section{- $\quad$ Le rendement des capitaux propres (ROE)}

Le rendement des capitaux propres peut être mesuré en utilisant le rapport entre le résultat net et le total des capitaux propres. Ce ratio permet d'appréhender la capacité de la banque à générer du profit à partir des apports des actionnaires. Le ROE représente le rendement des capitaux propres uniquement au moment de la clôture de l'exercice, c'est-à-dire en fin d'année. Donc, il ne prend pas en compte les variations pouvant intervenir au cours d'une même année. Afin de dépasser ce dysfonctionnement, nous avons opté dans notre étude pour une version ajustée du ROE qui est le ROAE (Return On Average Equity) représentant un rendement moyen des capitaux propres. A cet égard, la relation entre le ROE et la liquidité a été examinée dans différentes études dont certaines ont démontré que cette relation est négative et statistiquement significative du fait que les banques qui disposent d'un ratio de rendement des capitaux propres élevé ont la possibilité d'utiliser leurs revenus pour faire face à leurs engagements à court terme (Abdullah et Khan, 2012; Anam et al., 2012). Ainsi nous pouvons formuler l'hypothèse suivante :

\section{H3 : Le rendement des capitaux propres aura un impact négatif sur le risque de liquidité des banques islamiques.}

- $\quad$ Le rendement des actifs (ROA)

Le ROA représente le rendement des actifs et est mesuré par le rapport entre le résultat net et le total des actifs. Ce ratio donne une idée sur la capacité de la banque à générer du profit à partir de ses actifs. Pour la réalisation de notre étude, nous utiliserons également la version ajustée de ce ratio, à savoir le 
ROAA. En effet, plusieurs auteurs ont fait appel à ce ratio dans leur analyse des déterminants du risque de liquidité et certains ont démontré que le ROA a un impact positif et significatif sur le risque de liquidité du fait que les banques les plus rentables peuvent investir davantage et disposent donc d'une liquidité limitée, ce qui accroît leur risque de liquidité (Akhtar et al., 2011; Al-Harbi, 2020; Alzoubi, 2017; Ben Jedidia et Hamza, 2015; Boukhatem et Djelassi, 2020; Chowdhury et al., 2019; Gafrej et Boujelbene, 2017; Iqbal, 2012). De ce fait, l'hypothèse à vérifier est la suivante :

\section{H4 : Le rendement des actifs aura un impact positif sur le risque de liquidité des banques islamiques.}

\section{- $\quad$ La marge nette d'intérêt (NIM)}

La marge nette d'intérêt constitue également un ratio de performance permettant d'avoir une idée sur la rentabilité bancaire. Ce ratio est calculé en rapportant les revenus nets d'intérêt à l'actif productif, c'est-à-dire celui qui génère des intérêts. Ghenimi et Omri (2018) ont conclu que la marge nette d'intérêt a un impact significativement négatif sur le risque de liquidité en se basant sur le fait que la réalisation d'une marge nette importante peut aider la banque à faire face à ses dates d'échéances. En conséquence, la cinquième hypothèse se présente comme suit :

H5 : La marge nette d'intérêt aura un impact négatif sur le risque de liquidité des banques islamiques.

\subsubsection{La taille de la banque}

La relation entre la taille de la banque et la liquidité a été largement débattue dans la littérature financière. Selon l'hypothèse du « too big to fail », la taille d'une banque peut avoir un impact négatif sur sa liquidité et donc positif sur son risque de liquidité. Contrairement aux petites banques, certains établissements de crédit considèrent qu'elles sont suffisamment grandes pour ne pas faire faillite et sont donc moins motivées pour détenir des niveaux de liquidité élevés (Al-Harbi, 2020). De plus, une taille importante peut rendre délicate la gestion des risques au sein de la banque. A cet effet, de nombreux travaux de recherche ont approximé ce facteur par le logarithme naturel de l'actif total et ont conclu que la taille de la banque influence positivement son risque de liquidité (Anam et al., 2012; Boukhatem et Djelassi, 2020; Ghenimi et Omri, 2015, 2018; Iqbal, 2012; Ramzan et Zafar, 2014). Sur la base de l'analyse ci-dessus, la sixième hypothèse de notre étude est établie comme suit :

\section{H6 : La taille aura un impact positif sur le risque de liquidité des banques islamiques.}

\subsection{Les déterminants externes du risque de liquidité bancaire}

Outre les déterminants internes, cette étude examine également les déterminants externes du risque de liquidité bancaire et en particulier deux facteurs macroéconomiques au niveau des pays de la région MENA. Ces déterminants s'articulent principalement autour de la croissance économique et de l'inflation.

\subsubsection{La croissance économique}

La variable utilisée pour exprimer la croissance économique est le produit intérieur brut par habitant ou par tête (PIB/habitant). Ce dernier représente la valeur marchande totale de tous les biens et services finaux produits dans un pays à un moment donné (Alper et al., 2012). Il peut être considéré comme une mesure de la santé d'un pays et est également utilisé comme indicateur du cycle économique (Al-Harbi, 2017). Selon Yaacob et al. (2016), les banques préfèrent, en période de stabilité économique, détenir moins d'actifs liquides afin d'octroyer davantage de prêts et également en vue d'augmenter leurs 
investissements dans le but d'accroitre leur rentabilité. Une relation positive existe donc entre la croissance économique et le risque de liquidité bancaire. Ce constat a été corroboré par les résultats obtenus dans le cadre de plusieurs études ayant porté sur des banques islamiques dans différents contextes (Al-Harbi, 2020; Ghenimi et Omri, 2015). De ce fait, la septième hypothèse de notre étude se présente comme suit :

\section{H7 : La croissance économique aura un impact positif sur le risque de liquidité des banques islamiques.}

\subsubsection{L'inflation}

Le taux d'inflation peut être défini comme étant l'augmentation générale des niveaux de prix. L'impact du taux d'inflation sur la liquidité bancaire a fait l'objet de nombreux débats dans la littérature économique et financière. Ghenimi et Omri (2015) ont étudié les déterminants du risque de liquidité et ont soulevé que l'inflation avait un impact positif et statistiquement significatif sur le risque de liquidité. En effet, un taux d'inflation élevé peut être la cause d'une diminution du pouvoir d'achat des individus qui auront besoin de plus de fonds pour se procurer les mêmes biens, ce qui peut causer une augmentation de la demande des prêts, se traduisant ainsi par une diminution de la liquidité bancaire, d'où un accroissement du risque de liquidité (Trenca et al., 2015). En se basant sur l'analyse ci-dessus, la huitième hypothèse de notre étude est la suivante :

\section{H8 : L'inflation aura un impact positif sur le risque de liquidité des banques islamiques.}

\section{Méthodologie de recherche}

\subsection{Choix de l'échantillon}

Nous avons retenu dans notre étude 62 banques de 10 pays appartenant à la région MENA. Les données bancaires sont collectées à partir de la base des données de BankFocus (ex BankScope) et les données macroécomiques sont recueillies à partir des indicateurs publiés sur le site web de la Banque Mondiale.

\subsection{Spécification du modèle}

En nous basant sur les différents travaux empiriques qui se sont intéressés aux déterminants du risque de liquidité au niveau des banques islamiques, nous proposons une analyse de données de panel dynamique non cylindré. Ainsi, la spécification du modèle empirique est la suivante :

$\mathrm{Y}_{\mathrm{it}}=\alpha+\beta_{\mathrm{i}} \mathrm{X}_{\mathrm{it}}+\varepsilon_{\mathrm{it}}$

Où :

$\mathrm{Y}_{\mathrm{it}}$ représente les variables dépendantes $; \alpha$ représente la constante ou le terme fixe $; \beta_{i}(\mathrm{i}=1 \ldots \ldots 62)$ forme les coefficients de régression; $\mathrm{X}_{\mathrm{it}}$ symbolise l'ensemble des variables explicatives; i représente les banques de l'échantillon $(\mathrm{i}=1 \ldots . .62)$ qui sont observées sur une période de neuf ans s'étalant entre 2011 et $2019(\mathrm{t}=1 \ldots . .9) ; \varepsilon_{i t}$ indique le terme d'erreur.

Ainsi, notre analyse stipule que le risque de liquidité est une fonction du ratio d'adéquation du capital (CAR), du rendement des actifs (ROA), du rendement des capitaux propres (ROE), de la marge nette d'intérêt (NIM), du ratio des prêts non performants (NPL), de la taille de la banque, du taux de croissance (PIB), de l'inflation ainsi que de la variable retardée du risque de liquidité. Nous pouvons donc écrire nos équations sous la forme suivante : 


$$
\begin{aligned}
& \mathrm{L} 1=\alpha_{0}+\alpha_{1} \mathrm{~L}_{\mathrm{it}-1}+\alpha_{2} \mathrm{CAR}_{\mathrm{it}}+\alpha_{3} \mathrm{ROA}_{\mathrm{it}}+\alpha_{4} \mathrm{ROE}_{\mathrm{it}}+\alpha_{5} \mathrm{NIM}_{\mathrm{it}}+\alpha_{6} \mathrm{NPL}_{\mathrm{it}}+\alpha_{7} \text { Taille }_{\text {it }}+ \\
& \alpha_{8} \mathrm{PIB}_{\text {it }}+\alpha_{9} \mathrm{INF}_{\mathrm{it}} \\
& \mathrm{L} 2=\beta_{0}+\beta_{1} \mathrm{~L} 2_{\mathrm{it}-1}+\beta_{2} \mathrm{CAR}_{\mathrm{it}}+\beta_{3} \mathrm{ROA}_{\mathrm{it}}+\beta_{4} \mathrm{ROE}_{\mathrm{it}}+\beta_{5} \mathrm{NIM}_{\mathrm{it}}+\beta_{6} \mathrm{NPL}_{\mathrm{it}}+\beta_{7} \text { Taille }_{\mathrm{it}}+ \\
& \beta_{8} \mathrm{PIB}_{\text {it }}+\beta_{9} \mathrm{INF}_{\mathrm{it}}
\end{aligned}
$$

Où :

$\mathbf{L 1}$ représente le risque de liquidité à long terme ; et $\mathbf{L} \mathbf{2}$ représente le risque de liquidité à court terme.

\subsection{Mesure des variables}

- La variable dépendante : le risque de liquidité

Dans la modélisation conçue pour cette étude, nous avons mobilisé deux variables dépendantes :

- Le risque de liquidité à long terme (L1) : celui-ci est approximé par le ratio des prêts rapportés au total des actifs. En effet, ce ratio mesure le pourcentage de l'actif total que la banque a investi dans le financement. En général, les prêts sont moins liquides que les autres actifs. Ainsi, plus ce ratio est élevé, moins la banque dispose de liquidité (Ben Jedidia et Hamza, 2015).

- Le risque de liquidité à court terme (L2) : Afin de vérifier la robustesse de nos estimations, nous avons rajouté une deuxième variable dépendante notée L2 et approximée par le rapport entre la trésorerie et le total des actifs. Ce ratio renvoie au matelas de liquidité que la banque conserve comme ressources inutilisées et donne une idée sur la proportion des liquidités disponibles (Akhtar et al., 2011; Iqbal, 2012; Muharam et Kurnia, 2012).

\section{- Les variables explicatives}

Dans notre étude, le risque de liquidité constitue la variable à expliquer et ce en ayant recours à plusieurs variables explicatives, que nous récapitulons au niveau du tableau ci-après :

Tableau 1 : Variables explicatives

\begin{tabular}{|l|l|c|}
\hline Variables & \multicolumn{1}{|c|}{ Proxies } & Hypothèses \\
\hline ROE & Résultat net / Capitaux propres & $(-)$ \\
\hline ROA & Résultat net / Total Actif & $(+)$ \\
\hline NIM & Revenus nets d'intérêt /Actif Productif & $(-)$ \\
\hline CAR & (Tier 1 +Tier 2) / Actifs pondérés par les risques & $(-)$ \\
\hline NPL & Prêts non performants / Prêts bruts & $(+)$ \\
\hline Taille & Ln (Total Actif) & $(+)$ \\
\hline PIB & PIB par habitant & $(+)$ \\
\hline Inflation & Taux d'inflation & $(+)$ \\
\hline
\end{tabular}

\section{Résultats et discussions}

Après avoir présenté les modèles à étudier et les différentes variables qui le constituent, nous allons, à présent, analyser les résultats obtenus et ce en deux temps : nous allons, d'une part, effectuer une 
analyse descriptive basée essentiellement sur les statistiques descriptives et d'autre part, nous allons procéder à une analyse économétrique en faisant appel à la méthode des moments généralisés (GMM).

\subsection{Analyse descriptive}

\subsubsection{Statistiques descriptives}

Le tableau 2 montre que le ratio prêts/total des actifs (L1) moyen de notre échantillon est de $56.91 \%$ avec un écart-type de $16.82 \%$. Quant au ratio de trésorerie (L2) moyen, il est de $25.08 \%$ avec un écarttype de $11.2 \%$. Ces pourcentages élevés indiquent que les banques islamiques conservent des niveaux importants de liquidité afin de faire face à leur risque de liquidité. La valeur moyenne du rendement des capitaux propres (ROE) est de $8.3 \%$ mais sa dispersion est très élevée $(41.04 \%)$. Le rendement des actifs (ROA) a enregistré une moyenne assez faible de $0.82 \%$ avec une dispersion réduite autour de la moyenne de $2.71 \%$. En ce qui est des ratios NIM et NPL, ceux-ci enregistrent respectivement une moyenne de $2.92 \%$ et $8.59 \%$ avec un écart-type respectif de $3.84 \%$ et $11.71 \%$. Cette analyse descriptive fait également ressortir que le ratio CAR affiche une moyenne de $18.04 \%$ et une valeur de déviation de 0.1564 . Concernant la variable de la taille, la valeur moyenne calculée est de 8.45 avec une valeur de déviation de 1.6. En ce qui concerne les variables macroéconomiques, le taux d'inflation a enregistré une moyenne de $8 \%$ avec un écart-type de $10.56 \%$ tandis que la valeur moyenne du PIB est de $-0.07 \%$ avec une valeur de déviation de 0.039 .

Tableau 2 : Statistiques descriptives des variables

\begin{tabular}{lcccc}
\hline & Moyenne & Ecart-type & Maximum & Minimum \\
\hline L1 & 0.5691 & 0.1682 & 0.9471 & 0.0041 \\
L2 & 0.2508 & 0.1120 & 0.8725 & 0.0048 \\
ROE & 0.0830 & 0.4104 & 1.6814 & -7.1442 \\
ROA & 0.0082 & 0.0271 & 0.0783 & -0.3114 \\
NIM & 0.0292 & 0.0384 & 0.2102 & -0.3605 \\
CAR & 0.1804 & 0.1564 & 2.0441 & -0.2900 \\
NPL & 0.0859 & 0.1171 & 0.8320 & 0.0004 \\
Taille & 8.4522 & 1.6007 & 11.5369 & 2.6803 \\
PIB & -0.0007 & 0.0390 & 0.1187 & -0.0855 \\
Inflation & 0.0800 & 0.1056 & 0.3991 & -0.0209 \\
\hline Sourc: & & & &
\end{tabular}

Source : Etabli par l'auteur.

\subsubsection{Corrélation entre les variables}

Nous allons présenter dans les tableaux 3 et 4, ci-après, les coefficients de corrélation entre les variables dépendantes et l'ensemble des variables explicatives mobilisées. En effet, l'analyse de la matrice de corrélation de Pearson rend compte de l'importance de la corrélation entre chacune des variables dépendantes et les variables explicatives et permet ainsi d'apprécier le problème de multicolinéarité entre les variables. Nous pouvons considérer qu'il existe un problème de multicolinéarité lorsque le coefficient de corrélation est supérieur à $80 \%$. Le tableau 3 montre que plusieurs variables sont corrélées, mais pas au-delà du seuil critique de multicolinéarité. Ainsi, le coefficient de corrélation le plus élevé se situe entre L1 et le ratio NPL avec une corrélation négative de 
-0.281. Une corrélation négative est également observée entre L1 et le ROE et entre le PIB et l'inflation. En revanche, la variable dépendante L1 est positivement corrélée à la taille, au ROA, au NIM et au ratio CAR.

Tableau 3 : Matrice de corrélation de la variable dépendante L1

\begin{tabular}{|l|lllllllll|}
\cline { 2 - 8 } \multicolumn{1}{c|}{} & L1 & ROE & ROA & NIM & CAR & NPL & Taille & PIB & Inflation \\
\hline L1 & 1 & & & & & & & & \\
ROE & $-0,069$ & 1 & & & & & & & \\
ROA & 0,036 & 0,213 & 1 & & & & & & \\
NIM & 0,060 & $-0,041$ & 0,015 & 1 & & & & & \\
CAR & 0,057 & $-0,021$ & $-0,034$ & 0,026 & 1 & & & & \\
NPL & $-0,281$ & $-0,039$ & $-0,155$ & 0,049 & $-0,090$ & 1 & & & \\
Taille & 0,166 & 0,059 & 0,124 & $-0,237$ & 0,031 & $-0,101$ & 1 & & \\
PIB & $-0,063$ & $-0,051$ & $-0,054$ & 0,003 & 0,005 & $-0,026$ & $-0,028$ & 1 & \\
Inflation & $-0,002$ & 0,035 & 0,038 & $-0,184$ & $-0,121$ & 0,198 & 0,017 & $-0,095$ & 1 \\
\hline
\end{tabular}

Source : Etabli par l'auteur.

Quant au tableau 4 représentant les coefficients de corrélation entre la variable dépendante L2 et l'ensemble des variables explicatives, celui-ci indique qu'il existe une faible corrélation entre ces variables. Ainsi, la variable dépendante L2 est négativement corrélée aux ratios NIM, CAR et à l'inflation, tandis qu'elle est positivement corrélée aux variables explicatives restantes (ROE, ROA, NPL, la taille, PIB).

Tableau 4 : Matrice de corrélation de la variable dépendante L2

\begin{tabular}{|l|lllllllll|}
\cline { 2 - 8 } \multicolumn{1}{c|}{} & L2 & ROE & ROA & NIM & CAR & NPL & Taille & PIB & Inflation \\
\hline L2 & 1 & & & & & & & & \\
ROE & 0,076 & 1 & & & & & & & \\
ROA & 0,075 & 0,213 & 1 & & & & & & \\
NIM & $-0,032$ & $-0,041$ & 0,015 & 1 & & & & & \\
CAR & $-0,053$ & $-0,021$ & $-0,034$ & 0,026 & 1 & & & & \\
NPL & 0,052 & $-0,039$ & $-0,155$ & 0,049 & $-0,090$ & 1 & & & \\
Taille & 0,025 & 0,059 & 0,124 & $-0,237$ & 0,031 & $-0,101$ & 1 & & \\
PIB & 0,115 & $-0,051$ & $-0,054$ & 0,003 & 0,005 & $-0,026$ & $-0,028$ & 1 & \\
Inflation & $-0,088$ & 0,035 & 0,038 & $-0,184$ & $-0,121$ & 0,198 & 0,017 & $-0,095$ & 1 \\
\hline
\end{tabular}

Source : Etabli par l'auteur.

\subsection{Analyse économétrique}

Dans le but de comprendre la relation qui existe entre le risque de liquidité et les différents facteurs présentés ci-dessus, qu'ils soient internes ou externes à la banque, nous avons constaté que les 
méthodes économétriques classiques (méthode des moindres carrés ordinaires (MCO) par exemple) ne sont pas adaptées pour la réalisation d'une telle étude, car ne permettant pas d'obtenir des estimations représentatives et non biaisées en présence d'un problème d'endogénéité.

Le biais d'endogénéité renvoie à la corrélation qui existe entre une ou plusieurs variables explicatives et le terme d'erreur, dont les sources sont :

- Les variables omises : ce biais est lié à l'absence de variables qui sont supposées faire partie des variables explicatives mais qui ne le sont pas pour différentes raisons (une base de données incomplète pour notre cas). Ces variables omises sont alors contenues dans le terme d'erreur ;

- La simultanéité : c'est un biais qui se réalise lorsqu'il est difficile de statuer si c'est la variable indépendante qui détermine la variable dépendante ou l'inverse ;

- Les erreurs de mesure : c'est un biais qui concerne les erreurs qui peuvent se produire suite au choix des proxies à utiliser pour la quantification des variables étudiées. Toute différence entre la vraie valeur de la variable et celle que nous choisissons pour l'approximer est comprise dans le terme d'erreur.

Afin surmonter ce problème et en raison des considérations justifiées ci-dessous, nous avons opté pour la méthode des moments généralisés (GMM) sur panel dynamique non cylindré, dont les fondateurs sont Arellano et Bond (1991). En vue d'examiner l'efficacité de l'estimateur GMM en présence d'un panel dynamique, deux tests peuvent être envisagés : (1) Le test de suridentification de Sargan/Hansen qui examine si les variables retardées sont valides comme instruments ; (2) Les tests d'absence d'autocorrélation sérielle des termes d'erreur de premier et de second ordre AR (1) et AR (2) d'Arellano et Bond où l'hypothèse nulle indique l'absence d'autocorrélation des termes d'erreur. Pour appliquer la méthode GMM dans le cadre de la présente étude, nous avons fait appel au logiciel STATA et plus précisément au procédé XTABOND2. De plus, et dans le but d'obtenir la meilleure spécification de notre modèle, nous en avons testé plusieurs en nous basant sur différentes hypothèses quant à l'endogénéité des variables explicatives. La variable retardée du risque de liquidité est instrumentée par son propre retard en niveau et en différence (t-1) et les résultats obtenus sont présentés dans le tableau ci-après : 
Tableau 5 : Résultats empiriques

\begin{tabular}{|l|l|l|}
\cline { 2 - 3 } \multicolumn{1}{c|}{} & \multicolumn{1}{c|}{ L1 } & \multicolumn{1}{c|}{ L2 } \\
\hline CAR & $0.7171^{*} * * /(0.0470)$ & $0.2558 * * /(0.0601)$ \\
\hline NIM & $0.3646 /(0.5483)$ & $0.1283 * * /(0.0616)$ \\
\hline NPL & $-0.4972 * * * /(0.1169)$ & $0.0731 /(0.1338)$ \\
\hline ROA & $-0.2932 * * * /(0.0488)$ & $-0.0013 /(0.0466)$ \\
\hline ROE & $0.5286 * * /(0.2181)$ & $-0.9211 * * * /(0.2323)$ \\
\hline Taille & $-0.0054 /(0.0055)$ & $0.0018 /(0.0059)$ \\
\hline Inflation & $-0.0327 * * * /(0.0079)$ & $0.0201 * * /(0.0088)$ \\
\hline PIB & $-0.1181 * * /(0.0480)$ & $0.1133 * * /(0.0478)$ \\
\hline C & $0.0507 /(0.0848)$ & $-0.1248 /(0.0898)$ \\
\hline Observations & $0.4869^{*} * /(0.0754)$ & $-0.0271 /(0.0884)$ \\
\hline Sargan statistic & 372 & 371 \\
\hline P-value Sargan statistic & 0.30 & 11.79 \\
\hline AR (1) & -6.37 & 0.11 \\
\hline P-value AR (1) & 0.00 & -5.76 \\
\hline AR (2) & 1.48 & 0.00 \\
\hline P-value AR (2) & 0.14 & -2.97 \\
\hline & & 0.10 \\
\hline
\end{tabular}

Le test de suridentification de Sargan affiche une valeur de 67.37 pour la variable dépendante L1 et une valeur de 11.79 pour L2 avec des p-values respectives de 0.30 et 0.11 appartenant à l'intervalle [0.10_0.99], ce qui ne permet pas de rejeter l'hypothèse nulle de validité des restrictions de suridentification. La validité des instruments utilisés est ainsi confirmée, ce qui signifie la validité de nos résultats. En outre, les tests d'absence d'autocorrélation d'Arellano et Bond de premier et de second ordre relatifs à L1 affichent des p-values inférieures au seuil de 0.1 , ce qui signifie que nous rejetons l'hypothèse d'absence d'autocorrélation de premier ordre, mais que nous ne pouvons rejeter celle de second ordre d'Arellano et Bond. Cette conclusion est valable pour la variable dépendante L2 du fait que les p-values afférentes aux tests d'absence d'autocorrélation de premier et de second ordre la concernant sont de 0.00 et 0.10 respectivement. L'introduction de la valeur retardée des deux variables endogènes à droite de nos équations initiales implique l'existence d'un ajustement de liquidité. Selon le tableau 5, le coefficient d'ajustement de liquidité est statistiquement significatif au seuil de confiance de $99 \%$ avec un signe positif aussi bien pour L1 que pour L2.

En ce qui concerne la variable endogène L1, les résultats montrent que les variables explicatives NIM, NPL et la taille sont négatives et statistiquement significatives à un seuil de confiance de 99\%. L'inflation affiche également un signe négatif et statistiquement significatif au seuil de confiance de $95 \%$. Le ratio ROA a par contre une influence positive et statistiquement significative à un seuil de confiance de $95 \%$.

Le signe négatif affiché par le ratio de la marge nette d'intérêt indique qu'une augmentation de celui-ci est associée à une diminution de l'exposition des banques islamiques au risque de liquidité à long terme. En se référant au coefficient obtenu, nous constatons qu'une augmentation du ratio NIM de $1 \%$ implique une baisse du risque de liquidité à long terme de $0.4972 \%$, ce qui coöncide avec le résultat obtenu par Ghenimi et Omri (2018). La relation négative démontrée signifie que les banques islamiques 
peuvent, grâce à cette marge, faire face à leurs dates d'échéances ce qui réduit considérablement leur exposition au risque de liquidité. Notre cinquième hypothèse est ainsi confirmée.

Cependant, notre première hypothèse a été rejetée puisque les résultats affichent une relation négative entre le risque de liquidité à long terme et le risque de crédit que représente le ratio des prêts non performants NPL. Ainsi, une augmentation de $1 \%$ de ce dernier entraine une diminution du risque de liquidité à long terme de $0.2932 \%$. Ce résultat corrobore celui d'Iqbal (2012), Ghenimi et Omri (2015), Shamas et al. (2018), Chowdhury et al. (2019) et Al Harbi (2020). En effet, cette relation négative entre le ratio NPL et le risque de liquidité est fondée sur le fait qu'une mauvaise qualité des actifs peut se traduire par une rentabilité moindre et une faible liquidité d'où une exposition plus élevée au risque de liquidité (Shamas et al., 2018).

En ce qui est du signe positif affiché par le ratio ROA, celui-ci indique qu'une augmentation du rendement des actifs implique un accroissement de l'exposition des banques islamiques au risque de liquidité à long terme. Selon le coefficient obtenu, nous constatons qu'une augmentation du ROA de $1 \%$ implique un accroissement du risque de liquidité à long terme de $0.5286 \%$. Ce résultat coïncide avec celui d'Akhtar et al. (2011), Iqbal (2012), Gafrej et Boujelbene (2017), Hamza et Ben Jedidia (2015), Alzoubi (2017), Al Harbi (2020), Chowdhury et al. (2019) ainsi que celui de Boukhatem et Djelassi (2020). Cette relation positive signifie que les banques les plus rentables ont la possibilité d'investir davantage et disposent donc d'une liquidité limitée, ce qui accroit leur risque de liquidité. Ainsi, notre quatrième hypothèse est confirmée.

Quant à la taille de la banque islamique, les résultats infirment notre sixième hypothèse qui prévoit une relation positive avec le risque de liquidité. Ainsi, lorsque la taille de la banque augmente de 1\%, la variable dépendante $\mathrm{L} 1$ enregistre une baisse de $0.0327 \%$. Ce résultat a été corroboré au niveau d'autres travaux de recherche dont les auteurs ont conclu que lorsque la taille de la banque est importante, celleci est généralement plus stable et attire davantage de dépôts, en comparaison avec une banque de taille réduite (Abdullah et Khan, 2012; Chowdhury et al., 2019; Gafrej et Boujelbene, 2017). De plus, les grandes banques bénéficient d'un taux de supervision plus élevé leur permettant de réduire leur exposition au risque de liquidité (Zaghdoudi et Hakimi, 2017). Par ailleurs, les résultats démontrent que l'inflation influence négativement le risque de liquidité à long terme, ce qui indique que ce dernier augmente lorsque le taux d'inflation diminue. En effet, une augmentation du taux d'inflation de 1\% implique une baisse du risque de liquidité à long terme de $0.1181 \%$. Plusieurs études confirment ce constat en invoquant que dans un contexte inflationniste, le taux d'intérêt ou de profit a tendance à augmenter et peut donc réduire le niveau des financements octroyés par la banque ce qui implique une augmentation de la liquidité disponible au niveau de la banque, et par conséquent une réduction de l'exposition au risque de liquidité (Al-Harbi, 2020; Incekara et Cetinkaya, 2019; Yaacob et al., 2016). Notre huitième hypothèse n'est donc pas vérifiée.

En ce qui concerne la variable endogène $\mathrm{L} 2$, les résultats montrent que les variables explicatives CAR, inflation et taille de la banque sont positives et statistiquement significatives à un seuil de confiance de $95 \%$, alors que le ROA affiche un signe négatif et statistiquement significatif au seuil de confiance de $99 \%$.

Le ratio d'adéquation des fonds propres (CAR) est positivement lié au risque de liquidité à court terme de telle sorte que lorsque ce ratio augmente de $1 \%$, la variable dépendante L2 augmente de $0.1283 \%$. Ce constat est en adéquation avec les conclusions d'Iqbal (2012), Ghenimi et Omri (2015, 2018), Effendi et Disman (2017), Zaghdoudi et Hakimi (2017), Chowdhury et al (2019) et celles d'Al 
Harbi (2020). En effet, le coût d'opportunité occasionné par la disponibilité de ces fonds propres peut être le déclencheur d'une accentuation du risque de liquidité. Notre deuxième hypothèse prévoyant qu'il existe une relation positive entre le ratio d'adéquation des fonds propres et le risque de liquidité est ainsi rejetée. Les résultats montrent également que l'inflation agit positivement sur la variable endogène L2, c'est-à-dire que lorsque le taux d'inflation augmente de $1 \%$ le risque de liquidité à court terme enregistre une hausse de $0.1133 \%$. Ce constat a été confirmé par les travaux de recherche de Ghenimi et Omri (2015), où il a été soulevé qu'un taux d'inflation élevé peut être la cause d'une diminution du pouvoir d'achat des individus, qui auront besoin de plus de fonds pour se procurer les mêmes biens. En conséquence, la demande des prêts augmenterait, se traduisant ainsi par une diminution de la liquidité bancaire, d'où un accroissement du risque de liquidité (Ghenimi et Omri, 2015). De plus, la hausse du taux d'inflation influence également l'épargne, d'où une baisse en termes de dépôts qui se traduit également par une accentuation du risque de liquidité. Nous déduisons donc que le résultat obtenu confirme notre huitième hypothèse.

En ce qui est du signe négatif affiché par le ratio ROA, celui-ci indique qu'une augmentation du rendement des actifs implique une diminution de l'exposition des banques islamiques au risque de liquidité à court terme. En se référant au coefficient enregistré, nous constatons qu'une augmentation du ROA de $1 \%$ implique une diminution du risque de liquidité de $0.9211 \%$. Ce résultat coïncide avec celui de Muharam et Kurnia (2012) et Ghenimi et Omri (2015). Un tel lien négatif signifie qu'un ROA élevé indique que la banque parvient à générer, grâce à ses actifs, des revenus lui permettant de faire face à ses dates d'échéance. Ainsi, notre quatrième hypothèse qui suppose une relation positive entre le risque de liquidité et le rendement des actifs est rejetée. Par ailleurs, les résultats confirment notre sixième hypothèse qui prévoit une relation positive entre le risque de liquidité et la taille de la banque. Ainsi, lorsque la taille de la banque augmente de $1 \%$, la variable dépendante L2 augmente de $0.0201 \%$. Ce résultat a été confirmé par plusieurs travaux de recherche dont les auteurs ont conclu que plus la taille de la banque est importante, plus la gestion du risque de liquidité devient complexe et contraignante, contrairement aux petites banques où le niveau du risque de liquidité demeure relativement maitrisable (Al-Harbi, 2020; Boukhatem et Djelassi, 2020; Ghenimi et Omri, 2015, 2018; Iqbal, 2012; Ramzan et Zafar, 2014).

\section{Conclusion}

Les résultats de notre étude, appliquée à des données de panel et portant sur un échantillon de 62 banques islamiques de la région MENA, démontrent que le risque de liquidité inhérent à ces institutions peut être influencé par plusieurs facteurs. Nous avons ainsi soulevé que le ratio représentant le risque de liquidité à long terme a été négativement et de façon significative influencé par le risque de crédit, la marge nette d'intérêt, la taille de la banque et l'inflation, tandis qu'un impact positif a été exercé par le ratio du rendement des actifs. Nous avons aussi constaté que le ratio d'adéquation des fonds propres, l'inflation et la taille de la banque influencent positivement et significativement le risque de liquidité à court terme au sein des banques islamiques étudiées. Ce dernier a, par ailleurs, été influencé de façon négative par le ratio du rendement des actifs.

Il en ressort donc, que pour une meilleure maitrise de ce risque, que ce soit à long ou à court terme, il est essentiel de prendre en considération ces différents facteurs à tous les niveaux de la décision au sein de la banque islamique. En effet, au niveau stratégique, il serait souhaitable que ces facteurs soient intégrés en amont aux politiques de gestion du risque de liquidité afin d'assurer leur contrôle et leur suivi. De la même manière, ces déterminants doivent également être pris en compte au niveau 
opérationnel afin de permettre une mise en œuvre optimale de plans d'action qui soient en adéquation avec la vision stratégique globale de chacune des banques islamiques.

\section{BIBLIOGRAPHIE}

[1] Abdullah, A., and Khan, A.Q. (2012). Liquidity Risk Management: A Comparative Study between Domestic and Foreign Banks in Pakistan. Journal of Managerial Sciences 6, 62-72.

[2] Abdullah, A., and Khan, A.Q. (2012). Liquidity Risk Management: A Comparative Study between Domestic and Foreign Banks in Pakistan. Journal of Managerial Sciences 6, 62-72.

[3] Akhtar, M.F., Ali, K., and Sadaqat, S. (2011). Liquidity Risk Management: A comparative Study between Conventional and Islamic Banks of Pakistan. Interdisciplinary Journal of Research in Business 1, 35-44.

[4] Al-Harbi, A. (2017). Determinants of Banks Liquidity: Evidence from OIC Countries. Journal of Economic and Administrative Sciences 33, 164-177.

[5] Al-Harbi, A. (2020). Determinates of Islamic Banks Liquidity. Journal of Islamic Accounting and Business Research $11,1619-1632$.

[6] Al-Muharrami, S., and Hardy, D.C. (2013). Cooperative and Islamic Banks: What can they Learn from Each Other? International Monetary Fund WP/13/184.

[7] Alper, K., Hulagu, T., and Keles, G. (2012). An Empirical Study on Liquidity and Bank Lending (Research and Monetary Policy Department, Central Bank of the Republic of Turkey).

[8] Alzoubi, T. (2017). Determinants of Liquidity Risk in Islamic Banks. Banks and Bank Systems 12, $142-148$.

[9] Anam, S., Bin Hassan, S., Huda, H., Uddin, A., and Hossain, M. (2012). Liquidity Risk Management : A Comparative study between conventional and islamic banks of Bangladesh. Research Journal of Economics, Business and ICT 5.

[10] Ben Jedidia, K., and Hamza, H. (2015). Determinants of Liquidity Risk in Islamic Banks : A Panel Study. European Journal of Business and Management 7, 137-146.

[11] Boukhatem, J., and Djelassi, M. (2020). Liquidity Risk in the Saudi Banking System: Is There any Islamic Banking Specificity? Quarterly Review of Economics and Finance 77, 206-219.

[12] Chowdhury, M.M., Zaman, S., and Alam, M.A. (2019). Liquidity Risk Management of Islamic Banks in Bangladesh. International Journal of Business and Technopreneurship 9, 37-48.

[13] Diamond, D.W., and Dybvig, P.H. (1983). Bank Runs, Deposit Insurance, and Liquidity. The Journal of Political Economy 91, 401-419.

[14] Effendi, K.A., and Disman, D. (2017). Liquidity Risk: Comparison between Islamic and Conventional Banking. European Research Studies 20, 308-318.

[15] Gafrej, O., and Boujelbene, M. (2017). Liquidity Determinants of Islamic and Conventional Banks. Journal of Islamic Banking and Finance 34, 44-61.

[16] Ghenimi, A., and Omri, M. (2015). Liquidity Risk Management: A Comparative Study Between Islamic and Conventional Banks. Arabian Journal of Business and Management Review 3, 25-30.

[17] Ghenimi, A., and Omri, M. (2018). Les déterminants du risque de liquidité dans les banques islamiques : Cas de la région MENA. La Revue Gestion et Organisation 10, 127-136.

[18] Greenwood, J., and Jovanovic, B. (1990). Financial Development, Growth, and the Distribution of Income. Journal of Political Economy 98, 1076-1107.

[19] Hassoune, A. (2003). La solvabilité des banques islamiques : forces et faiblesses. Revue d'économie Financière 72 , $277-297$.

[20] Hesse, H., Jobst, A., and Solé, J. (2008). Trends and Challenges in Islamic Finance. World Economics 9, 175-193.

[21] Incekara, A., and Cetinkaya, H. (2019). Liquidity Risk Management : A Comparative Analysis of Panel Data Between Islamic and Conventional Banking in Turkey. Procedia Computer Science 158, 955-963.

[22] Iqbal, A. (2012). Liquidity Risk Management: A Comparative Study between Conventional and Islamic of Pakistan. Global Journal of Management and Business Research 12, 54-64.

[23] Ismal, R. (2010). The Management of Liquidity Risk in Islamic Banks : The Case of Indonesia. Durham University. 
[24] Khan, T., and Ahmed, H. (2001). Risk Management: An Analysis of Issues in Islamic Financial Industry (Djeddah KSA: Islamic Development Bank, Islamic Research and Training Institute).

[25] Menicucci, E., and Paolucci, G. (2016). The Determinants of Bank Profitability : Empirical Evidence from European Banking Sector. Journal of Financial Reporting and Accounting 14, 86-115.

[26] Mennawi, A.N.A., and Ahmed, A.A. (2020). The Determinants of Liquidity Risk in Islamic Banks: A Case of Sudanese Banking Sector. International Journal of Islamic Banking and Finance Research 4, 38-49.

[27] Mohammad, S., Asutay, M., Dixon, R., and Platonova, E. (2020). Liquidity Risk Exposure and its Determinants in the Banking Sector: A Comparative Analysis Between Islamic, Conventional and Hybrid banks. Journal of International Financial Markets, Institutions and Money 66.

[28] Muharam, H., and Kurnia, H.P. (2012). The influence of fundamental factors to liquidity risk on banking industry Comparative study between Islamic bank and Conventional bank in Indonesia. In Conference in Business, Accounting and Management (CBAM), pp. 359-368.

[29] Ramzan, M., and Zafar, M.I.Z. (2014). Liquidity Risk Management in Islamic Banks : A Study of Islamic Banks of Pakistan. Interdisciplinary Journal of Contemporary Research in Business 5, 199-216.

[30] Repullo, R. (2004). Capital Requirements, Market Power, and Risk Taking in Banking. Journal of Financial Intermediation 13, 156-182.

[31] Shamas, G.S., Zairani, Z., and Zairy, Z. (2018). The Impact of Bank’s Determinants on Liquidity Risk : Evidence from Islamic Banks in Bahrain. Journal of Business and Management 6, 1-22.

[32] Syed, S. (2013). State of Liquidity Management in Islamic Financial Institutions. Islamic Economic Studies 21, $63-98$.

[33] Trenca, I., Petria, N., and Corovei, E.A. (2015). Impact of Macroeconomic Variables upon the Banking System Liquidity. Procedia Economics and Finance 32, 1170-1177.

[34] Yaacob, S.F., Abdul Rahman, A., and Abdul Karim, Z. (2016). The Determinants of Liquidity Risk : A Panel Study of Islamic Banks in Malaysia. Journal of Contemporary Issues and Thought 6, 73-82.

[35] Zaghdoudi, K., and Hakimi, A. (2017). The Determinants of Liquidity Risk : Evidence from Tunisian Banks. Journal of Applied Finance and Banking 7, 71-81. 